Old Dominion University

ODU Digital Commons

Physics Faculty Publications

Physics

2011

\title{
Electronically Enhanced Surface Diffusion During Ge Growth on $\mathrm{Si}(100)$
}

\author{
Ali Orguz Er \\ Old Dominion University \\ Hani E. Elsayed-Ali \\ Old Dominion University, helsayed@odu.edu
}

Follow this and additional works at: https://digitalcommons.odu.edu/physics_fac_pubs

Part of the Atomic, Molecular and Optical Physics Commons, Electronic Devices and Semiconductor Manufacturing Commons, and the Semiconductor and Optical Materials Commons

\section{Repository Citation}

Er, Ali Orguz and Elsayed-Ali, Hani E., "Electronically Enhanced Surface Diffusion During Ge Growth on Si(100)" (2011). Physics Faculty Publications. 25.

https://digitalcommons.odu.edu/physics_fac_pubs/25

\section{Original Publication Citation}

Er, A. O., \& Elsayed-Ali, H. E. (2011). Electronically enhanced surface diffusion during Ge growth on Si(100). Journal of Applied Physics, 109(8), 084320. doi:10.1063/1.3567918 


\title{
Electronically enhanced surface diffusion during Ge growth on $\mathrm{Si}(100)$
}

\author{
Ali Oguz $\mathrm{Er}^{1}$ and Hani E. Elsayed-Ali ${ }^{2, a)}$ \\ ${ }^{1}$ Department of Physics, Old Dominion University, Norfolk, Virginia 23529, USA \\ ${ }^{2}$ Department of Electrical and Computer Engineering and the Applied Research Center, \\ Old Dominion University, Norfolk, Virginia 23529, USA
}

(Received 22 November 2010; accepted 17 February 2011; published online 20 April 2011)

\begin{abstract}
The effect of nanosecond pulsed laser excitation on surface diffusion during the growth of Ge on $\mathrm{Si}(100)$ at $250^{\circ} \mathrm{C}$ was studied. In situ reflection high-energy electron diffraction was used to measure the surface diffusion coefficient while ex situ atomic force microscopy was used to probe the structure and morphology of the grown quantum dots. The results show that laser excitation of the substrate increases the surface diffusion during the growth of $\mathrm{Ge}$ on $\mathrm{Si}(100)$, changes the growth morphology, improves the crystalline structure of the grown quantum dots, and decreases their size distribution. A purely electronic mechanism of enhanced surface diffusion of the deposited Ge is proposed. @ 2011 American Institute of Physics. [doi:10.1063/1.3567918]
\end{abstract}

\section{INTRODUCTION}

Recent experiments have shown that electronic excitation of the substrate during growth can assist epitaxial growth, ${ }^{1-3}$ modify surface morphology, and reduce the size distribution of grown quantum dots (QDs), making them more uniform. ${ }^{1,4,5}$ Enhancement of the effective surface diffusion of adatoms by the excitation laser was proposed as a possible mechanism responsible for the observed effects. ${ }^{1,6}$

Surface diffusion is a critical parameter for epitaxial thin film growth since diffusion allows the adatoms to hop to the appropriate active lattice sites. For surface diffusion to occur, a certain amount of energy must be available to the adatoms. This energy can originate from the kinetic or the potential energy of the adsorbate or can be supplied thermally by substrate heating. The rate of surface diffusion depends on a variety of factors, including the energy of the incoming adsorbate, the strength of the surface-adatom bond, orientation of the surface lattice, densities of atomic steps, surface reconstruction, attraction and repulsion between surface species, and externally supplied energy. ${ }^{7}$ The surface diffusion coefficient $D_{s}$ is known to follow an Arrhenius form with temperature and is proportional to the vibrational energy in the reaction coordinate.

Several external energy sources applied to surfaces have been shown to increase surface diffusion. These include low energy ion bombardment and the resulting momentum transfer to the surface species, ${ }^{8,9}$ charge carrier injection, ${ }^{10}$ and light-induced migration of $\mathrm{Ag}$ atoms originating from electronic excitation. ${ }^{11}$

The diffusion of $\mathrm{Ge}$ adatoms on $\mathrm{Si}(100)-(2 \times 1)$ is highly anisotropic in nature with diffusion favored in a direction parallel to the dimer rows. ${ }^{12}$ Surface migration is at least 1000 times faster along the dimer rows than perpendicular to them. ${ }^{13}$ The surface diffusion coefficient of $\mathrm{Ge}$ atoms depends on the surface orientation. For example, Ge atoms have almost two times greater diffusion coefficient on

\footnotetext{
${ }^{\text {a) }}$ Author to whom correspondence should be addressed. Electronic mail: helsayed@odu.edu.
}

$\mathrm{Si}(100)$ than $\mathrm{Si}(111) .{ }^{14}$ The diffusion coefficient of Ge adatoms on $\mathrm{Si}(001)$ was found to be $D_{\mathrm{Ge}} \sim 2.53 \times 10^{-7}$ $\exp (-0.676 \pm 0.03 \mathrm{eV} / \mathrm{kT}) \mathrm{cm}^{2} / \mathrm{s}$ in the temperature range between $650-725^{\circ} \mathrm{C} .{ }^{15} \mathrm{We}$ report on the effect of laser substrate excitation on surface diffusion during the growth of $\mathrm{Ge}$ on $\mathrm{Si}(100)-(2 \times 1)$ by pulsed laser deposition. Our results show that applying the excitation laser during the growth increases the surface diffusion coefficient in a way that is exponentially dependent on laser intensity.

The surface diffusion coefficient was measured using reflection high-energy electron diffraction (RHEED) intensity recovery after the growth interruption. The intensity of the RHEED diffraction spots depends on the surface structure and morphology. Increased surface roughness reduces the diffraction spot intensities. Therefore, immediately after starting the Ge growth by ablating the Ge target, the intensity of the RHEED diffraction orders decreases. For epitaxial growth, the RHEED intensity can recover when the growth is interrupted. This intensity recovery time depends on the amount of time it takes the surface species to rearrange to their final locations. RHEED recovery upon deposition termination can generally be described by a two-exponential decay, ${ }^{16} I=A_{0}-A_{1} \exp \left(-t / \tau_{1}\right)-A_{2} \exp \left(-t / \tau_{2}\right)$, where $A_{1}$ and $A_{2}$ are usually positive constants and $\tau_{1}$ and $\tau_{2}$ are temperature-dependent time constants of the fast and slow stages, respectively. These time constants could be explained as diffusion along a step edge and diffusion across the terrace for fast and slow recoveries, respectively. The fast processes are generally attributed to smoothening of the front growth profile, while slow processes are attributed to long-range order reactions such as rearrangement of terraces and/or the reduction of one-dimensional disorder. ${ }^{16,17}$ It is often observed, as in the present study, that the RHEED recovery curve can be well described by a one exponential with time constant $\tau .{ }^{16}$ For a vicinal surface, the surface diffusion coefficient $D_{s}$ is related to the average terrace width $L$ by $D_{s} \sim$ $L^{2} / \tau$, where $D_{s}$ is the diffusion coefficient and $\tau$ is the relaxation time constant which depends on the density of nucleation sites and diffusion velocity. ${ }^{16,18}$ 


\section{EXPERIMENT}

The $\operatorname{Si}(100)$ substrates ( $p$-type boron doped, resistivity $0.060-0.075 \Omega \mathrm{cm}$, miscut angle $0.38^{\circ}$ ) were chemically etched by using a modified Shiraki method before being loaded into the UHV chamber (base pressure $\sim 7.0 \times 10^{-10}$ Torr). The Ge target was an undoped $n$-type with a resistivity of 45-58.7 $\Omega \mathrm{cm}$. The $\mathrm{Si}(100)$ sample was degassed at $\sim 700^{\circ} \mathrm{C}$ in UHV for $24 \mathrm{~h}$ and then flashed to $\sim 1200^{\circ} \mathrm{C}$ for $\sim 60$ s to obtain the $(2 \times 1)$ reconstruction. The substrates were heated by direct current flow. The surface temperature was measured using a combination of a chromel-alumel (Ktype) thermocouple that was mechanically attached to the substrate surface and a pyrometer. A Q-switched Nd:YAG (wavelength $\lambda=1064 \mathrm{~nm}$, full width at half maximum (FWHM) of $\sim 40 \mathrm{~ns}, 10 \mathrm{~Hz}$ repetition rate) was split into an ablation beam and an excitation beam of nonequal powers by means of a half wave-plate and a thin film polarizing beam splitter. The $p$-polarized ablation beam was focused on the rotating Ge target to a spot size $\sim 400 \mu \mathrm{m}$ (measured at $1 /$ e of the peak value), resulting in a laser energy density of $\sim 5 \mathrm{~J} / \mathrm{cm}^{2}$. The $s$-polarized excitation beam was left unfocused with a beam diameter of $\sim 6.0 \mathrm{~mm}$ (measured at $1 / \mathrm{e}$ peak value) and was used to irradiate the sample surface. Both the ablation and the excitation laser beams were incident on the Ge target and $\mathrm{Si}$ substrate at $45^{\circ}$. Sample-to-target distance was $\sim 8 \mathrm{~cm}$. A $20-\mathrm{keV}$ well-collimated RHEED electron gun with a spot size less than $90 \mu \mathrm{m}$ in diameter was used to monitor the growth, while a partially coated phosphor screen displayed the electron diffraction pattern, which was recorded by means of charge-coupled device camera. The electron beam had a grazing angle $\sim 2.5 \pm 0.5^{\circ}$ with the $\mathrm{Si}(100)$ surface. The final film thickness measurement was done by a spectroscopic ellipsometer with an accuracy of \pm 1 ML. Post deposition tapping-mode atomic force microscope (AFM) was used to study the morphology of the film.

\section{RESULTS AND DISCUSSIONS}

We have previously studied the effect of the excitation laser on the $\mathrm{Ge}$ growth on $\mathrm{Si}(100)-(2 \times 1)$ at a substrate temperature of $\sim 250{ }^{\circ} \mathrm{C}$. ${ }^{1}$ For samples grown below $390^{\circ} \mathrm{C}$, the intensity of the $\mathrm{Si}(100)-(2 \times 1)$ RHEED spots was observed to decay continuously with deposition time until they disappear, resulting in a diffused pattern. ${ }^{1,19}$ This indicates the formation of three-dimensional (3D) structures that collectively lack long-range order, as was confirmed by RHEED and AFM measurements. The AFM image obtained at $250{ }^{\circ} \mathrm{C}$ showed a collection of $3 \mathrm{D}$ clusters with different shapes and sizes. ${ }^{1}$

In the present study, the substrate temperature was $\sim 250{ }^{\circ} \mathrm{C}$ and the ablation laser energy density was $5 \mathrm{~J} / \mathrm{cm}^{2}$, while the excitation laser energy density was varied. Figure 1 shows a set of RHEED patterns of Ge QDs grown on $\operatorname{Si}(100)-(2 \times 1)$ with different excitation laser energy densities applied to the substrate. For Figs. 1(a) through 1(f), the RHEED patterns were obtained after 8000 laser pulses. Postdeposition analysis of the Ge film thickness using an ellipsometer confirmed that the films had a thickness of $22 \mathrm{ML}$.
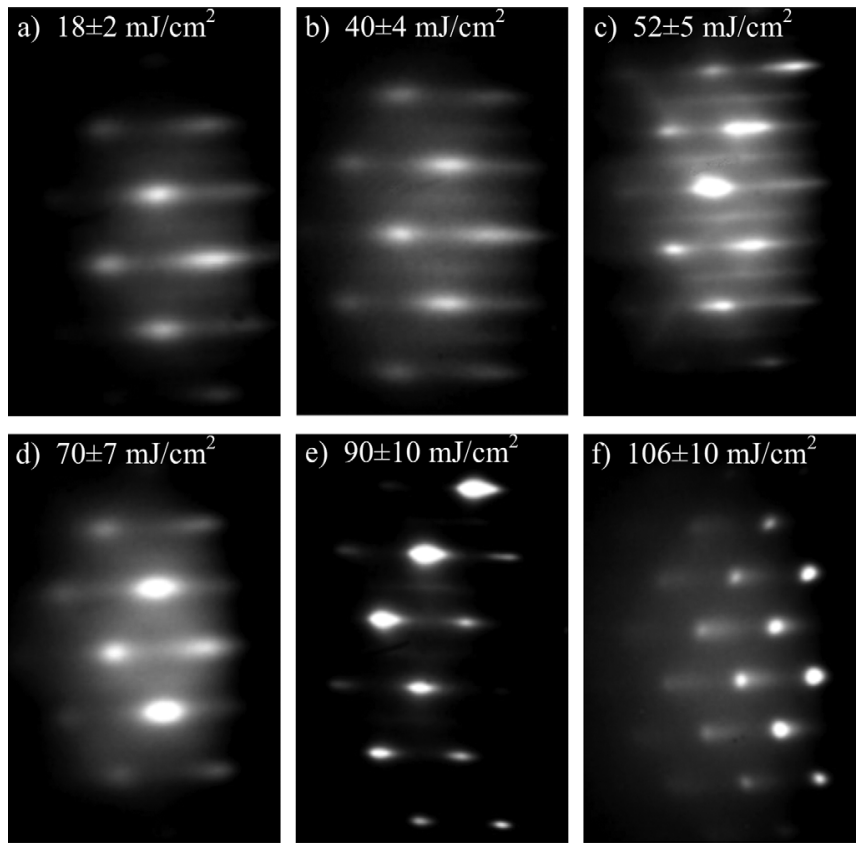

FIG. 1. RHEED patterns after deposition of $22 \mathrm{ML}$ Ge for a substrate temperature of $250^{\circ}$ C. $20 \mathrm{keV}$ electron beam energy, angle of incidence with the surface $\sim 2.5^{\circ}$, and ablation laser energy density of $5 \mathrm{~J} / \mathrm{cm}^{2}$ is used in all experiments. The excitation laser energy density is (a) $18 \pm 2 \mathrm{~mJ} / \mathrm{cm}^{2}$, (b) $40 \pm 4 \mathrm{~mJ} / \mathrm{cm}^{2}$, (c) $52 \pm 5 \mathrm{~mJ} / \mathrm{cm}^{2}$, (d) $70 \pm 7 \mathrm{~mJ} / \mathrm{cm}^{2}$, (e) $90 \pm 10 \mathrm{~mJ} / \mathrm{cm}^{2}$, and (f) $106 \pm 10 \mathrm{~mJ} / \mathrm{cm}^{2}$.

The corresponding AFM images of these films are shown in Figs. 2(a) though 2(f).

\section{A. Results}

For an excitation laser energy density of $18 \pm 2 \mathrm{~mJ} / \mathrm{cm}^{2}$, the RHEED image in Fig. 1(a) shows a elongated transmission pattern, indicating 3D growth. The AFM image consists of high-density Ge islands, most of which are rectangular-based or square-based islands with some small fraction of domes. An individual island is shown in the inset of Fig. 2(a). The density of islands is $\sim 7.5 \pm 1.5 \times 10^{9} \mathrm{~cm}^{-2}$. The mean length, $l$, is $57 \pm 28 \mathrm{~nm}$. The mean height is $\sim 9 \pm 4 \mathrm{~nm}$.

We next increased the excitation laser to $40 \pm 4 \mathrm{~mJ} / \mathrm{cm}^{2}$ while all other conditions remained as in Fig. 1(a). The observed RHEED pattern, shown in Fig. 1(b), does not change significantly from that in Fig. 1(a). However, the island density and coverage ratio decreased, while the mean height and length increased, as observed by analysis of the AFM images. In Fig. 2(b), Ge islands with different shapes and sizes are visible. A single island at this condition could be seen in the inset of Fig. 2(b). Those islands mainly consist of rectangular-based and square-based pyramid-shape islands while the fraction of domes was increased. The shape of the islands becomes more uniform. The average island size increased when compared to the conditions used in Fig. 2(a). This is consistent with the coalescence of the small islands to larger ones. The island density decreased to $5.0 \pm 1.0 \times 10^{9} \mathrm{~cm}^{-2}$. The mean length also increased to $60 \pm 30 \mathrm{~nm}$ with an average height of $\sim 13 \pm 5 \mathrm{~nm}$.

When the laser energy density was increased to $52 \pm 5$ $\mathrm{mJ} / \mathrm{cm}^{2}$, the RHEED streaks upon growth termination shown 

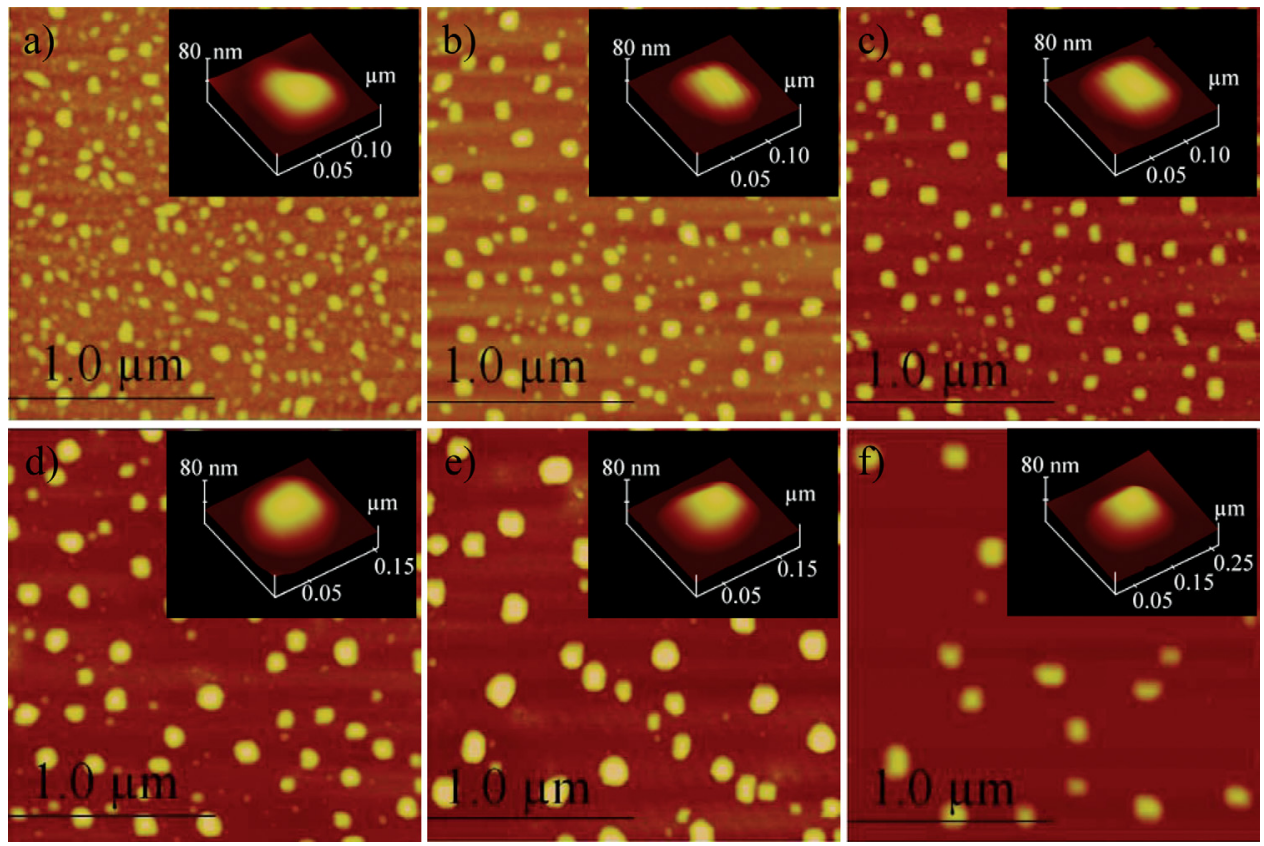

FIG. 2. (Color online) The AFM images of the individual islands with excitation energy density of (a) $18 \pm 2 \mathrm{~mJ} / \mathrm{cm}^{2}$, (b) $40 \pm 4 \mathrm{~mJ} / \mathrm{cm}^{2}$, (c) $52 \pm 5 \mathrm{~mJ} / \mathrm{cm}^{2}$, (d) $70 \pm 7 \mathrm{~mJ} / \mathrm{cm}^{2}$, (e) $90 \pm 10 \mathrm{~mJ} / \mathrm{cm}^{2}$, and (f) $106 \pm 10 \mathrm{~mJ} / \mathrm{cm}^{2}$. Increasing excitation laser energy density causes island density and the lateral aspect ratio and coverage ratio to decrease, while the average area, length, and height of the islands increase and the size distribution become narrower. Insets show individual islands for each condition.

in Fig. 1(c), are less elongated and become slightly more intense than in Figs. 1(a) and 1(b). The corresponding AFM image shows an increase in the size of the islands while their lateral aspect ratio becomes smaller. The island density is $\sim 3.6 \pm 0.8 \times 10^{9} \mathrm{~cm}^{-2}$ while the mean length and height are $\sim 75 \pm 27 \mathrm{~nm}$ and $\sim 16 \pm 4 \mathrm{~nm}$, respectively. One of the individual islands is shown in the inset of Fig. 2(c).

This trend continued as the excitation energy density was increased to $70 \pm 7 \mathrm{~mJ} / \mathrm{cm}^{2}$. In Fig. 1(d) the elongated RHEED transmission pattern becomes more rounded showing a spotty transmission pattern not falling on Laue zones, indicating the growth of 3D islands. A single island is visible in the AFM image in the inset of Fig. 2(d). It is seen that the lateral aspect ratios for those islands are getting smaller with the increase of the excitation laser energy density while the mean length and height increase. In Fig. 2(d), the island density is $\sim 2.5 \pm 0.5 \times 10^{9} \mathrm{~cm}^{-2}$; the mean length for those islands is $\sim 90 \pm 40 \mathrm{~nm}$; and the mean height is $\sim 21 \pm 7 \mathrm{~nm}$.

For an excitation laser energy density of $90 \pm 10 \mathrm{~mJ} /$ $\mathrm{cm}^{2}$, the final RHEED pattern shown in Fig. 1(e), develops an additional transmission pattern compared to Fig. 1(d), indicative of better QD crystalline structure. The islands mainly consist of dome-shaped and rectangular based islands. The corresponding AFM image shows that the island density is $1.5 \pm 0.5 \times 10^{9} \mathrm{~cm}^{-2}$, the mean length is $110 \pm 40$ $\mathrm{nm}$, and the mean height is $29 \pm 9 \mathrm{~nm}$. Some individual islands, mainly consisting of dome-shaped islands, are seen in Fig. 2(e).

Finally, the excitation laser energy density was increased to $106 \pm 10 \mathrm{~mJ} / \mathrm{cm}^{2}$. In this case, the final RHEED image becomes brighter with a well-defined spotty pattern and shows more transmission pattern, indicating new facet formation and increased crystalline quality, as shown in Fig. 1(f). The resulting AFM image, shown in Fig. 2(f), shows that the island density is further reduced to $0.8 \pm 0.3 \times 10^{9} \mathrm{~cm}^{-2}$, the mean length increased to $131 \pm 33$ $\mathrm{nm}$, and the mean height became $\sim 33 \pm 7 \mathrm{~nm}$. The islands became more uniform in size and shape and developed mainly dome shapes. These results show that increasing the excitation laser energy from $18 \pm 2 \mathrm{~mJ} / \mathrm{cm}^{2}$ to $106 \pm 10 \mathrm{~mJ} /$ $\mathrm{cm}^{2}$ at a substrate temperature of $250{ }^{\circ} \mathrm{C}$ causes the Ge island density, lateral aspect ratio, and coverage ratio to decrease, while the average area, length, and height of the islands increase and become more uniform in size and shape. The development of the RHEED patterns and associated Ge quantum dot morphology with the increase in the laser excitation energy density is analogous to what is observed when the substrate temperature is raised during deposition. ${ }^{1}$ Raising the substrate temperature is known to increase surface diffusion.

To investigate the effect of electronic excitation on surface diffusion, we observed the RHEED intensity recovery of the specular spot upon growth termination. The characteristic relaxation time constant is obtained from the RHEED intensity recovery curve. ${ }^{17,18,20}$ The surface diffusion coefficient $D_{s}$ is estimated from $D_{s} \sim L^{2} / \tau$, where $\tau$ is the experimentally measured RHEED relaxation time upon growth termination and $L$ is the average terrace length of the vicinal surface.

Figure 3 shows the specular spot RHEED intensity recovery curves obtained after growth interruption subsequent to 8000 laser deposition pulses. The recovery curves follow a simple exponential growth with a time constant $\tau=65 \pm 7$ for an excitation laser of $18 \pm 2 \mathrm{~mJ} / \mathrm{cm}^{2}$. Taking the average terrace width $L=20 \mathrm{~nm}$, based on the vicinal angle of the $\mathrm{Si}(100)$ substrate, a diffusion coefficient $D_{s} \sim 6 \times 10^{-14}$ $\mathrm{cm}^{2} / \mathrm{s}$ is obtained. It was previously found that the temperature dependence of the Ge adatom diffusion coefficient on $\mathrm{Si}(100)$ could be approximated by $D_{\mathrm{Si}(100)}=3.3 \times 10^{-2}$ $\exp (-1.2 \mathrm{eV} / \mathrm{kT}) \mathrm{cm}^{2} / \mathrm{s}^{14}$ At a substrate temperature of $250{ }^{\circ} \mathrm{C}$ without any excitation, this approximation gives $D_{s}$ $\sim 9 \times 10^{-14} \mathrm{~cm}^{2} / \mathrm{s}$. We were not able to measure $D_{s}$ at $250{ }^{\circ} \mathrm{C}$ without applying the excitation laser, since there is no specular spot at the end of the deposition. 


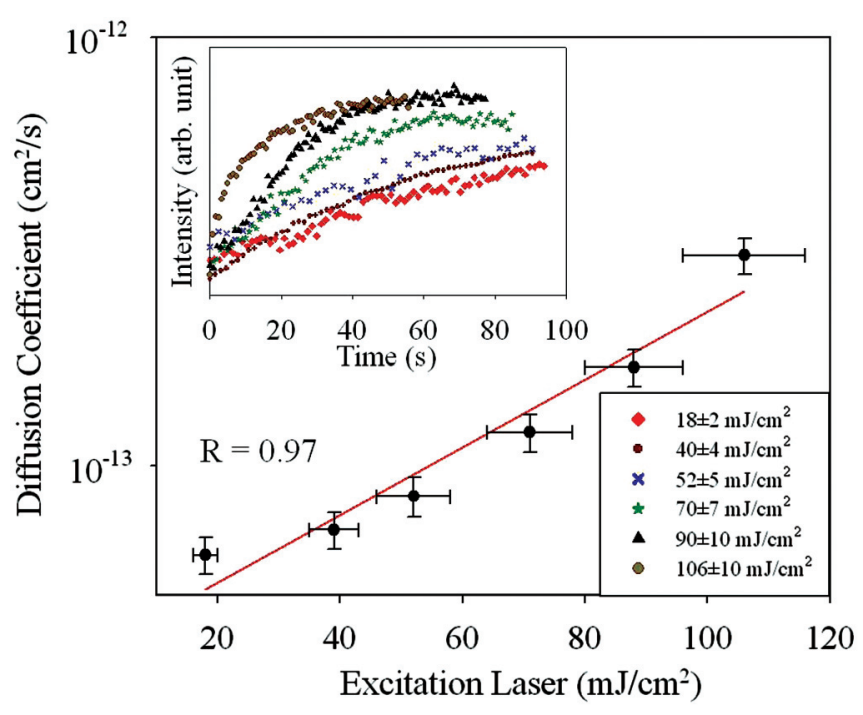

FIG. 3. (Color online) The surface diffusion coefficient versus excitation laser energy density after 8000 deposition pulses (22 ML). The inset shows the RHEED recovery curves obtained following the growth interruption at different excitation laser energy densities. The surface diffusion coefficient increases with the increasing excitation laser energy density.

\section{B. Discussions}

Applying an excitation laser results in epitaxial film growth, allowing the measurement of $D_{s}$. We should note that we are measuring the diffusion of $\mathrm{Ge}$ on the Ge wetting layer. It was shown that Ge atoms move faster than $\mathrm{Si}$ atoms on $\mathrm{Ge}(111)$ and the diffusion length increased by a factor of almost 10 when the substrate was changed from Si to Ge. ${ }^{21}$ Unlike Ge on $\mathrm{Si}(100)$, the diffusion of $\mathrm{Ge}$ on $\mathrm{Si}(001)$ covered by $1 \mathrm{ML}$ of Ge shows nearly isotropic behavior and it has a lower diffusion coefficient. ${ }^{22}$ With the increase in the excitation laser energy density, the RHEED intensity recovery time $\tau$ was observed to decrease and, therefore, the measured value of $D_{s}$ was increased, as shown in the inset of Fig. 3 . Applying the excitation laser during the growth noticeably increases $D_{s}$. A fit of the recovery curve in Fig. 3 gives the dependence of the surface diffusion coefficient on the excitation laser energy density $I$ in $\mathrm{mJ} / \mathrm{cm}^{2}$ such that $D_{s}=5.8+0.28^{*} \exp (0.04 I)$, where $D_{s}$ is in $10^{-14} \mathrm{~cm}^{2} / \mathrm{s}$. The correlation coefficient $R$ was found to be 0.97 from linear regression. For a substrate temperature of $250{ }^{\circ} \mathrm{C}$ and a laser excitation energy density of $106 \pm 10 \mathrm{~mJ} / \mathrm{cm}^{2}, \tau$ was $13 \pm 2 \mathrm{~s}$ corresponding to $D_{s}=3.1 \pm 0.3 \times 10^{-13} \mathrm{~cm}^{2} / \mathrm{s}$.

The value of $D_{s}$ obtained from the exponential increase of the specular RHEED intensity upon growth termination does not distinguish the diffusion of adatoms, small islands and clusters, and the migration of atoms from formed islands. Since we observe a one exponential for the RHEED intensity recovery, the recovery time $\tau$ does not distinguish between the various surface processes involved. Diffusion of islands and clusters strongly depends on their size and density. ${ }^{23}$ The motion of clusters may occur via displacement of individual atoms, sections of the clusters, or clusters moving as a whole. ${ }^{24}$ It was also shown that the mobility of small clusters decreases with increasing $\operatorname{size}^{25}$ and the diffusion coefficient of the islands has a power-law dependence on the island size. ${ }^{26}$ As the excitation laser energy density was increased, the island nucleation density was observed to decrease. This is consistent with an increase in $D_{s}$ with excitation.

The rise in surface temperature due to the laser pulse was calculated using a one-dimensional heat-diffusion model. According to this model, the maximum temperature rise due to absorption of the $1064 \mathrm{~nm}$ excitation laser in the skin depth of $\mathrm{Si}(\sim 60 \mu \mathrm{m})$ is $\sim 23^{\circ} \mathrm{C}$. The surface temperature drops to the substrate temperature by heat diffusion in $\sim 0.1 \mu \mathrm{s}$. For Ge, the skin depth for $1064 \mathrm{~nm}$ is $200 \mathrm{~nm}$, and the maximum temperature rise in this case is $\sim 200^{\circ} \mathrm{C}$ if irradiated with $106 \pm 10 \mathrm{~mJ} / \mathrm{cm}^{2}$, which is the maximum laser energy density used during this work. Heat diffusion to the Si substrate limits the surface temperature excursion time to $\sim 0.1 \mu \mathrm{s}$. The Ge atoms move just $\sim 1.6 \times 10^{-11} \mathrm{~cm}$ in $0.1 \mu \mathrm{s}$ at $250^{\circ} \mathrm{C}$, which is clearly too small to affect the nucleation and growth mechanism. Therefore, thermal effects can be excluded and the observed increase in surface diffusion is electronic in nature.

It is well known that an electron or a photon interacting with the surface can induce an electronic excitation causing the emission of ions or neutral particles. We can rule out the Knotek-Feibelman (KF) mechanism since KF requires photons with energies of tens of $\mathrm{eV} .^{27}$ The Menzel-Gomer and Redhead (MGR) model can also be ruled out since excitons on $\mathrm{Si}$ and $\mathrm{Ge}$ surfaces are not self-trapped and the applied photon energy is relatively low. ${ }^{28}$

Sumi proposed a two-hole localization (THL) model and applied it successfully to explain electronically induced bond rupture at $\mathrm{Si}$ and other surfaces. ${ }^{29}$ The primary assumption in THL is that surface bond rupture leading to neutral-atomic desorption can be induced by strong lattice relaxations associated with the localization of two valence holes on the same surface bond. The localization of the second hole causes strong vibrations of the surface atom, which could lead to bond breaking. These atoms could be ejected due to this transient strong lattice vibration (phonon kick) with a distribution of translational energies that starts from a given onset. ${ }^{30}$ Vibrational relaxation after electronic excitation would lead to many phonons being emitted. The lattice forms a continuum of motions that can absorb the energy of the vibrational relaxation. The bonded atoms vibrate more strongly as the energy of the surface atoms increases. If the phonon kick perpendicular to the surface imparted to a surface atom is not sufficient to cause desorption, the enhanced vibrational motion could lead to increased surface diffusions.

A possible scenario involves electron-hole generation in the substrate followed by hole diffusion to the surface and two-hole localization. ${ }^{29} \mathrm{Yu}$ and Tanimura investigated the laser-induced desorption of $\mathrm{Si}$ adatoms on $\mathrm{Si}(100)-(2 \times 1)$ when the surface is excited by $2.48 \mathrm{eV}$ laser pulses. ${ }^{31}$ Their results show that electronic excitation causes the desorption of $\mathrm{Si}$ atoms from a certain adatom configuration. The desorption yield was super-linearly dependent on the laser fluence and therefore, was consistent with the two-hole localization model. Desorption yields with successive laser pulses indicated that the adatom configuration that was reactive to desorption transformed by laser excitation into a different form that is less susceptible to desorption. The results show that 
free holes are more effectively trapped at surface-defect sites. This suggests that substrate excitation during deposition causes hole localization preferentially at adatom sites.

Once the Ge wetting layer is formed, two-hole localization can occur on the Ge surface. At $250{ }^{\circ} \mathrm{C}$, the indirect $\mathrm{Ge}$ energy bandgap is $0.57 \mathrm{eV}$ and $\mathrm{Ge}$ has a direct bandgap at $0.7 \mathrm{eV}$. The absorption coefficient of $1064 \mathrm{~nm}$ in $\mathrm{Ge}$ is $1.6 \times 10^{4} \mathrm{~cm}^{-1}$, leading to significantly higher electron-hole generation in the Ge wetting layer than the Si substrate. The hole density in Ge due to absorption of the laser pulse is $\sim 10^{23} \mathrm{~cm}^{-3}$. The surface hole density depends on many parameters that include surface recombination and diffusion across the $\mathrm{Si} / \mathrm{Ge}$ interface. We are not aware of any study done on electronic bond breaking on electronically excited Ge surfaces. However, in general, the two-hole localization mechanism, followed by the phonon-kick, is applicable to semiconductors. Energetically, this mechanism could be effective on Ge surfaces. For surface bond breaking, the phonon-kick has to transfer enough energy to the top atom along the bond direction to break that bond. If that energy transfer is not sufficient for bond breaking, then the atom will have a vibrational excitation that can lead to surface hopping. Therefore, THL on the Ge surface can lead to selective energy transfer to the Ge adatoms since these atoms constitute a defect site. The energy that is preferentially given to these adsorbed atoms can result in their hopping to epitaxially settle on the surface. The measurement of Ge film thickness for different excitation laser energy densities showed that the Ge coverage is not affected by the excitation laser; thus, we conclude that atom desorption by electronic excitation is too small for the studied conditions.

Our previous picosecond time-resolved RHEED studies of excitation of the three low-index surfaces of Ge with 1064 $\mathrm{nm}, 100 \mathrm{ps}$ laser pulses have conclusively shown that the surface temperature behaves as expected from a simple heat diffusion model. ${ }^{32-34}$ This observation rules out an electronic mechanism that transfers energy to surface atoms causing heating above that expected from simple laser absorption in the skin depth followed by heat diffusion. However, we point out that RHEED probes surface atoms over a very large area. The mechanism we are proposing for enhanced surface diffusion of the adsorbed Ge atoms is preferential to the adatoms, which only has very small coverage. Therefore, such preferential enhancement of surface hopping of the adatoms would not be sufficient to allow it to be detected through RHEED observation of the transient Debye-Waller factor. Time-resolved RHEED experiments with shorter time resolution, performed by Zewail et al., have shown that electronic excitation of semiconductor surfaces results in surface contraction followed by expansion. ${ }^{35}$ The initial structural changes (tens of ps) are due to nonthermal distortion of the binding forces due to electron-hole generation. These effects are dissipated in a few tens of ps and the surface then shows expansion as expected. ${ }^{35}$ Since the surface growth process is too slow compared to the detected bond softening time, we do not anticipate that such bond softening is directly involved in the observed nonthermal effects on QD growth. Dynamic TEM has been recently developed and used to image transient structures in thin films with 15 -ns temporal resolution. ${ }^{36-38}$
An extension of this technique to develop a dynamic reflection electron microscope $\left(\right.$ REM) ${ }^{39}$ could allow the real-time observation of the growth of the quantum dots and resolve how the growth dynamics is affected by electronic excitation.

\section{CONCLUSION}

We have studied the surface diffusion coefficient of Ge during pulsed laser deposition of $\mathrm{Ge}$ on $\mathrm{Si}(100)-(2 \times 1)$ with different excitation laser energy densities. Applying the excitation laser to the substrate during the growth increases the surface diffusion coefficient, changes the QD morphology and density, and improves the size uniformity of the grown quantum dots. We have ruled out thermal effects. A purely electronic mechanism of enhanced surface diffusion of $\mathrm{Ge}$ is proposed. The nature of this nonthermal mechanism could be due to two-hole localization followed by a phonon kick of the adsorbate. In order to determine the range of conditions for which electronic excitation is effective in the lowtemperature growth of epitaxial thin films and for enhancing surface diffusion, along with understanding the mechanism involved, future work will need to investigate the effect of the excitation wavelength and that of doping. The excitation wavelength is known to strongly affect the bond rupture at surfaces. For $\mathrm{Si}$, the bond rupture rate by $460 \mathrm{~nm}$ was found to be $\sim 100$ times more than that for $1064 \mathrm{~nm}$ at the same fluence. ${ }^{40}$ The bond rupture was resonantly enhanced at an excitation photon energy of $2 \mathrm{eV} .{ }^{41}$ However, the morphology of vacancy formation was the same regardless of the laser wavelength, which suggested that a similar mechanism of bond rupture was involved.

While the growth of the total count of vacancy clusters, irrespective of their size, is about the same for n-type and ptype surfaces, the total vacancy-site densities produced on $n$ type surfaces were significantly more than for p-type surfaces. The bond rupture at sites nearest to the pre-existing vacancies was significantly enhanced for n-type surfaces. ${ }^{40}$ Excited species generated in the bulk must localize at the surface to affect thin film growth. The transport process of carriers and its surface localization is strongly dependent on band bending. By performing experiments to compare excitation effects on n-type and p-type materials, band bending effects can be clarified.

Observation of surface diffusion at submonolayer coverage by atom tracking in a scanning tunneling microscope (STM) can be particularly important because this can provide an atomic view of how adsorbate motion is affected by electronic excitation. ${ }^{42,43}$ STM during growth can provide a series of images at regular scanning speed that are generally enough to get information about atom hopping and morphology evolution. The STM studies can also allow us to observe any surface defect generation and any changes in adsorption sites due to electronic excitation. These studies can be used to develop a better picture of the mechanism involved in electronically enhanced surface diffusion.

\section{ACKNOWLEDGMENTS}

This material is based upon work supported by the U.S. Department of Energy, Division of Material Science, under 
Grant No. DE-FG02-97ER45625 and the National Science Foundation under Grant Nos. DMR-9988669 and MRI0821180 .

${ }^{1}$ A. O. Er and H. E. Elsayed-Ali, J. Appl. Phys. 108, 034303 (2010).

${ }^{2}$ T. Inoue, Y. Yamamoto, and M. Satoh, J. Vac. Sci. Technol. A 19, 275 (2001).

${ }^{3}$ A. O. Er, W. Ren, and H. E. Elsayed-Ali, Appl. Phys. Lett. 98, 013108 (2011).

${ }^{4}$ A. V. Dvurechenskii, V. A. Zinov'ev, and Zh. V. Smagina, JETP Lett. 74, 267 (2001).

${ }^{5}$ V. A. Volodin, A. V. Yakimov, A. V. Dvurechenskiǔ, M. D. Efremov, I. Nikiforov, E. I. Gatskevich, G. D. Ivlev, and G. Y. Mikhalev, Semiconductors 40, 202 (2006).

${ }^{6} \mathrm{~N}$. Itoh and A. M. Stoneham, Materials Modification by Electronic Excitation (Cambridge University Press, Cambridge, 2001).

${ }^{7}$ D. L. Smith, Thin-Film Deposition: Principles \& Practice (McGraw-Hill, New York, 1995).

${ }^{8}$ W. Shindo and T. Ohmi, J. Appl. Phys. 79, 2347 (1996).

${ }^{9}$ R. Ditchfield and E. G. Seebauer, Phys. Rev. Lett. 82, 1185 (1999).

${ }^{10}$ G. Lengel, J. Harper, and M. Weimer, Phys. Rev. Lett. 76, 4725 (1996).

${ }^{11}$ R. Kometer and N. Schwentner, J. Chem. Phys. 104, 6967 (1996).

${ }^{12}$ D. Srivastava and B. J. Garrison, Phys. Rev. B 46, 1472 (1992).

${ }^{13}$ Y. -W. Mo and M. G. Lagally, Surf. Sci. 248, 313 (1991).

${ }^{14}$ A. Dolbak and B. Olshanetsky, Cent. Eur. J. Phys. 4, 310 (2006).

${ }^{15}$ H. J. Kim, Z. M. Zhao, J. Liu, V. Ozolins, J. Y. Chang, and Y. H. Xie, J. Appl. Phys. 95, 6065 (2004).

${ }^{16}$ M. A. Hafez and H. E. Elsayed-Ali, J. Appl. Phys. 101, 113515 (2007).

${ }^{17}$ B. A. Joyce, P. J. Dobson, J. H. Neave, and J. Zhang, Surf. Sci. 174, 1 (1986).

${ }^{18}$ J. H. Neave, P. J. Dobson, B. A. Joyce, and J. Zhang, Appl. Phys. Lett. 47, 100 (1985).

${ }^{19}$ M. S. Hegazy and H. E. Elsayed-Ali, J. Appl. Phys. 104, 124302 (2008).

${ }^{20}$ D. H. A. Blank, G. J. H. M. Rijnders, G. Koster, and H. Rogalla, Appl. Surf. Sci. 138-139, 17 (1999).

${ }^{21}$ V. Cherepanov and B. Voigtländer, Phys. Rev. B 69, 125331 (2004).

${ }^{22}$ R. Hull and J. C. Bean, Germanium Silicon: Physics and Materials (Academic, San Diego, 1999).
${ }^{23}$ L. Bardotti, P. Jensen, A. Hoareau, M. Treilleux, and B. Cabaud, Phys. Rev. Lett. 74, 4694 (1995).

${ }^{24}$ K. Oura, V. G. Lifshits, A. A. Saranin, A. V. Zotov, and M. Katayama, Surface Science: An Introduction (Springer, Berlin, 2003).

${ }^{25}$ J. M. Wen, S.-L. Chang, J. W. Burnett, J. W. Evans, and P. A. Thiel, Phys. Rev. Lett. 73, 2591 (1994).

${ }^{26}$ A. Bogicevic, S. Liu, J. Jacobsen, B. Lundqvist, and H. Metiu, Phys. Rev. B 57, R9459 (1998).

${ }^{27}$ Z. Wu, Phys. Lett. A 131, 486 (1988).

${ }^{28}$ J. Kanasaki, A. Okano, K. Ishikawa, Y. Nakai, and N. Itoh, Nucl. Instrum. Methods Phys. Res. B 101, 93 (1995).

${ }^{29}$ H. Sumi, Surf. Sci. 248, 382 (1991).

${ }^{30}$ J. Kanasaki, K. Iwata, and K. Tanimura, Phys. Rev. Lett. 82, 644 (1999).

${ }^{31}$ I.-K. Yu and K. Tanimura, Solid State Commun. 101, 429 (1997).

${ }^{32}$ X. Zeng and H. E. Elsayed-Ali, Surf. Sci. 497, 373 (2002).

${ }^{33}$ X. Zeng and H. E. Elsayed-Ali, Phys. Rev. B 64, 085410 (2001).

${ }^{34}$ X. Zeng, B. Lin, I. El-Kholy, and H. E. Elsayed-Ali, Phys. Rev. B 59, 14907 (1999).

${ }^{35}$ F. Vigliotti, S. Chen, C.-Y. Ruan, V. A. Lobastov, and A. H. Zewail, Angew. Chem., Int. Ed. 43, 2705 (2004).

${ }^{36}$ T. LaGrange, M. R. Armstrong, K. Boyden, C. G. Brown, G. H. Campbell, J. D. Colvin, W. J. DeHope, A. M. Frank, D. J. Gibson, F. V. Hartemann, J. S. Kim, W. E. King, B. J. Pyke, B. W. Reed, M. D. Shirk, R. M. Shuttlesworth, B. C. Stuart, B. R. Torralva, and N. D. Browning, Appl. Phys. Lett. 89, 044105 (2006).

${ }^{37}$ M. R. Armstrong, B. W. Reed, B. R. Torralva, and N. D. Browning, Appl. Phys. Lett. 90, 114101 (2007)

${ }^{38}$ J. S. Kim, T. LaGrange, B. W. Reed, M. L. Taheri, M. R. Armstrong, W. E. King, N. D. Browning, and G. H. Campbell, Science 321, 1472 (2008).

${ }^{39}$ Z. L. Wang, Reflection Electron Microscopy and Spectroscopy of Surfaces (Cambridge University Press, Cambridge, 1996).

${ }^{40}$ E. Inami and K. Tanimura, Phys. Rev. B 76, 035311 (2007).

${ }^{41}$ J. Kanasaki, T. Ishida, K. Ishikawa, and K. Tanimura, Phys. Rev. Lett. 80, 4080 (1998).

${ }^{42}$ B. S. Swartzentruber, Phys. Rev. Lett. 76, 459 (1996).

${ }^{43}$ M. C. Tringides and M. Hupalo, J. Phys.: Condens. Matter. 22, 264002 (2010). 